\title{
Correction to: Chinese Culture of Intelligence
}

\section{Correction to:}

K. Wang, Chinese Culture of Intelligence, https://doi.org/10.1007/978-981-13-3173-2

In the original version of the book, the funder information should be included in copyright page. The correction book has been updated with the change. 